\title{
A strategic model for epidemic control in aquaculture
}

\author{
Darren M. Green ${ }^{a}$
}

January 13, 2010

a Institute of Aquaculture, University of Stirling, Stirling, Stirlingshire FK9 4LA, UK. Tel: +44 1786 467872; Fax: +44 1786472133.

E-mail address:

darren.green@stir.ac.uk (D.M. Green).

\begin{abstract}
A stochastic metapopulation model of infectious disease was developed to model the spread of disease within and between sites of a region of an aquaculture industry. The study was a theoretical one examining the effect of transmission parameters through a sensitivity analysis. Production was modelled as either dispersed over many sites, or concentrated into small areas to provide 'firebreaks' between such areas as a disease control strategy. The effectiveness of such a control strategy could then be examined for different industry and disease parameters (for example, overall production, and rates of within- and between-site infection). At the within-site level, contact was modelled as either frequency or density dependent, either of these extreme formulations being potentially appropriate for different diseases. Under density dependence, the effect of high host density of increasing the basic reproduction number $R_{0}$ dominates, in contrast to the frequency dependent model. However, for both model types, concentration of production into separate areas successfully slows the spread of simulated disease, particularly where long-distance transmission of the pathogen is weak due to fast attenuation of infectious agent over distance and time.

Keywords: aquaculture; Monte Carlo; $R_{0}$; Gillespie algorithm
\end{abstract}




\section{Introduction}

As disease control measures to combat infections such as pancreas disease and infectious salmon anaemia in Atlantic salmon, governments have implemented such policies as control zones and restrictions on siting of aquaculture farms in both Norway and Scotland (Anon, 2008). With these measures, there has been a general tendency towards concentration of production into fewer populations with greater separation. In Scotland, the number of Atlantic salmon sites declined from 189 to 135 between 1999 and 2007, while production varied around c. 130,000 tonnes over the same period (Smith, 2008). In aggregating aquacultural production, the proposed benefit is that increased distance between sites acts as an epidemiological 'firebreak', preventing spread of disease from infected sites to susceptible sites. However, locally increasing host density has long been associated with risk of infectious disease (Reno, 1998). Aggregating production therefore carries with it both benefits and costs in terms of disease risk, and whether the benefits outweigh the costs is very likely to depend upon the features of particular diseases. This balance is the subject of this paper.

With production being divided into high-density sites, there are two main levels of epidemiological unit: the site, and the fish. The added complexity of sites containing structure due to having multiple pens, cages, ponds, or raceways is not considered in this paper. This two-level contact structure is easily implemented using a metapopulation model (Levins, 1969), where a population of individual fish (and their pathogens) exists at each of a population of sites (with flow of pathogen between sites). Metapopulation models are an established tool in modelling the spread of diseased individuals or infectious agent in such structured populations, for example for measles (Grenfell and Harwood, 1997) and bovine tuberculosis in possums (Fulford et al., 2002). At the between-site level, we are concerned with the relatively rare but important event of disease introduction. Modelling such events requires care.

Consider a disease with a risk of de novo infection of $10^{-5} y^{-1}$. Once introduced, the disease increases with an intrinsic rate of natural increase $r$ of $10 y^{-1}$. In a stochastic model, one would expect one infection event every 10,000 years. However, in a continuous, deterministic equivalent model, it takes approximately 5 years until a population size of one infected individual is reached. This is quite a bit faster. This simple model suffers badly from the 'atto-fox' problem $\left(10^{-18}\right.$ fox $)$ of allowing implausibly small proportions of a single individual to be simulated (Mollison, 1991). Clearly, the continuous, deterministic model is far from appropriate in modelling risk of disease under these circumstances, with rare - but important - events. The opposite problem is encountered in the Nicholson-Bailey model (Nicholson and Bailey, 1935) of host-parasitoid population dynamics. The deterministic form of the equation predicts ever-increasing oscillations in populations of both species. However, the troughs in these populations are ever smaller over time, and in an equivalent stochastic model extinction of one or other species is rapidly reached.

A stochastic modelling approach is more appropriate for modelling such risk of disease incursion, as has been carried out (in the field of aquaculture) for risk of inter-river transmission of Gyrodactylus salaris (Paisley et al., 1999; Høgåsen and Brun, 2003), and inter-site spread of infectious salmon anaemia (ISA; Scheel et al., 2007), and more generally by Thrush and Peeler (2006). These approaches are complemented by risk evaluation studies of low-frequency, high-impact disease transmission events, e.g. for infectious salmon anaemia (ISA; Munro et. al. 2003) and see Murray and Peeler (2005) and Peeler et al. (2007) for general principles.

In this paper, we propose a simple model for the spread of disease amongst a population of marine fish farms, using a metapopulation framework, which is fully 
stochastic. The model parameters include disease-related and production-related parameters, allowing predictions to be made strategically about the effects of aggregating or dispersing, increasing or decreasing, production, in terms of the risk and potential spread of different diseases. The study is a theoretical one, using hypothetical parameter values to make relative comparisons between model outputs through sensitivity analysis. This allows us to identify which parameters might be important for different types of diseases, and thus where experimental studies might be usefully focussed. The paper is not concerned with parameterising the model for specific diseases: this is a complex task in its own right.

Our model assumes that the force of infection between sites is related to distance, as might be the case where a substantial part of the risk of disease transmission between sites comes from spread of infectivity through the water column (either direct or by a vector), as can be the case for highly infectious diseases such as ISA (e.g.

Gustafson et al., 2007). However other potentially infectious links between sites are probable. Wellboat movements have been implicated as a potential disease transmission risk (Murray et al., 2002); and for ISA, one recent study indicated risk from sources aside from the local contact network and seaway to be a large component of risk of infection in the Norwegian salmon industry (Scheel et al., 2007). Though an important route of pathogen introduction, for salmon, site-to-site movements outside of stocking and and harvest are relatively infrequent (Green et al., 2009). Therefore, strategically, there is merit in considering the potential spread of epidemic diseases that operate over short timescales through local areas such as single management areas, outside of introduction through anthropogenic routes.

\section{Method}

\subsection{Model}

We begin with the following simple compartmental, differential equation model of density-dependent epidemic dynamics (i.e. per-individual contact is rate proportional to population size) and production at the level of an individual farm.

$$
\begin{aligned}
& \frac{d i}{d t}=\beta s i-\gamma i-\mu i \\
& \frac{d s}{d t}=-\beta s i-\mu s+\alpha
\end{aligned}
$$

All individuals, both susceptible $(s)$ and infectious ( $i$ ), are subject to harvesting at rate $\mu$ and there is a constant stocking of new susceptibles at rate $\alpha$. Susceptible individuals are infected at rate $\beta$, with density dependence, and infected individuals are removed at rate $\gamma$. There is no separate removed/recovered class. The basic reproduction number, $R_{0}$ is given simply by $R_{0}=\frac{s \beta}{\gamma+\mu}$, dependent on the population size at the point of introduction of disease. For $R_{0}>1$, introduction of disease leads to an epidemic, and $R_{0}<1$ no epidemic.

This model is extended to a metapopulation model by considering multiple sites, denoted by subscript $u$. The attenuating effect of distance upon the rate at which infected individuals at site $u$ can infect susceptibles at site $v$ is denoted by $\xi_{u, v}$, as a multiplier of the equivalent within-site infection rate. For simplicity of reading, we handle between-site infection separately to within-site infection, and set $\xi_{u, u}$ to zero everywhere below.

$$
\begin{aligned}
\frac{d i_{u}}{d t} & =\beta s_{u} i_{u}+\sum_{v=1}^{n} \xi_{v, u} \beta s_{u} i_{v}-\gamma i_{u}-\mu i_{u} \\
\frac{d s_{u}}{d t} & =-\beta s_{u} i_{u}-\sum_{v=1}^{n} \xi_{v, u} \beta s_{u} i_{v}+\alpha-\mu s_{u}
\end{aligned}
$$

If we sum over all sites (writing the sums of $i$ and $s$ as $I$ and $S$ ), then we obtain equations 
for the dynamics of the whole population:

$$
\begin{aligned}
& \frac{d I}{d t}=\beta \sum_{u=1}^{n} s_{u} i_{u}+\beta \sum_{u=1}^{n} s_{u} \sum_{v=1}^{n} \xi_{v, u} i_{v}-(\gamma+\mu) I \\
& \frac{d S}{d t}=-\beta \sum_{u=1}^{n} s_{u} i_{u}-\beta \sum_{u=1}^{n} s_{u} \sum_{v=1}^{n} \xi_{v, u} i_{v}+n \alpha-\mu S .
\end{aligned}
$$

From these equations, an expression for the basic reproduction ratio $R_{0}$ may be obtained. However, given density dependence, this will be dependent on $S$ as mentioned above. Therefore $s_{u}$ for each site is set at its disease-free equilibrium value of $\frac{\alpha}{\mu}$, obtained by setting $\beta=0$ and solving (1). Total stocking across the system of all sites is denoted by $A$, independent of the number of sites, and stocking distributed equally across all sites: $\alpha=\frac{A}{n}$. The numbers of infected individuals across all sites is assumed as equal and small $i_{u}=i \gtrsim 0$.

$$
\frac{d I}{d t}=\frac{\beta A}{n \mu} \sum_{u=1}^{n}\left[\frac{I}{n}+\sum_{v=1}^{n} \xi_{v, u} \frac{I}{n}\right]-(\gamma+\mu) I
$$

If the landscape is symmetrical with $\xi_{u, v}=\xi_{v, u}$, and homogeneous so that the summation $\sum_{v=1}^{n} \xi_{v, u}=\Xi_{u}$ is the same for all sites $u$, then we can write

$$
\frac{d I}{d t}=\frac{\beta A}{n \mu}[1+\Xi] I-(\gamma+\mu) I .
$$

Parameter $\Xi$ therefore represents the total force of infection posed by a site or experienced by a site as a multiplier of the within-site transmission rate $\beta$. By analogy with a standard $S I R$ model, taking the ratio of the recruitment and removal rates for the $I$ class, we can obtain the following expression for $R_{0}$ :

$$
R_{0}=\frac{\beta A(1+\Xi)}{n \mu(\gamma+\mu)}
$$

Alternatively, we can assume that all infection is initially located at a single site: $I=i_{j}$. In this case, we derive the following expression for $\frac{d I}{d t}$ :

$$
\begin{aligned}
\frac{d I}{d t} & =\beta s_{j} i_{j}+\beta \sum_{u=1}^{n} s_{u} \xi_{j, u} i_{j}-(\gamma+\mu) i_{j} \\
& =\frac{\beta A}{n \mu} I+\frac{\beta A}{n \mu} \sum_{u=1}^{n} \xi_{j, u} I-(\gamma+\mu) I .
\end{aligned}
$$

This expression simplifies to (5) above, and thus we obtain the same expression for $R_{0}$ by this method as above. The presence of $n$ in the $R_{0}$ equation indicates that by subdividing the population (increasing $n$ ), $R_{0}$ is quickly reduced, all other parameters being equal.

By setting the two derivatives $\frac{d S}{d t}$ and $\frac{d I}{d t}$ in equation (3) to zero, we can obtain expressions for the equilibrium sizes of the infectious and susceptible populations $\left(I_{\infty}\right.$ and $S_{\infty}$ ) where this is the same for all sites.

$$
\begin{aligned}
I_{\infty} & =\frac{A}{\gamma+\mu}-\frac{\mu n}{\beta(1+\Xi)} \\
S_{\infty} & =\frac{(\gamma+\mu) n}{\beta(1+\Xi)}=\frac{A}{\mu R_{0}}
\end{aligned}
$$

where $I_{\infty}$ only has a positive solution where $R_{0}>1 . I_{\infty}=0$ is a second, unstable, solution with the expected disease-free equilibrium value of $S_{\infty}=\frac{A}{\mu}$.

\subsection{Frequency dependence}

Alternatively, the above model could be formulated with frequency-dependent transmission within site (constant per-individual contact rate). Amending the single-patch model (1), one obtains

$$
\begin{aligned}
& \frac{d i}{d t}=\beta^{\prime} \frac{s i}{s+i}-\gamma i-\mu i \\
& \frac{d s}{d t}=-\beta^{\prime} \frac{s i}{s+i}-\mu s+\alpha
\end{aligned}
$$

where $R_{0}=\frac{\beta^{\prime}}{\gamma+\mu}$, independent of $s$. The prime mark indicates that this is not the same parameter $\beta$ as in the density-dependent model; nor does it have the same units. For the multiple-site model, frequency dependence is applied only to the within-site dynamics: (2) is amended 
replacing the term $i$ with $\frac{i}{s+i}$ as follows:

$$
\begin{aligned}
& \frac{d i_{u}}{d t}=\beta s_{u} \frac{i_{u} \rho_{\beta}}{s_{u}+i_{u}}+\sum_{v=1}^{n} \xi_{v, u} \beta s_{u} i_{v}-\gamma i_{u}-\mu i_{u} \\
& \frac{d s_{u}}{d t}=-\beta s_{u} \frac{i_{u} \rho_{\beta}}{s_{u}+i_{u}}-\sum_{v=1}^{n} \xi_{v, u} \beta s_{u} i_{v}+\alpha-\mu s_{u} .
\end{aligned}
$$

The parameter $\rho_{\beta}$ indicates the population size at which density-dependent and -independent transmission become equivalent, and is necessary to keep the terms of the equation dimensionally compatible $\left(\beta^{\prime}=\beta \rho_{\beta}\right)$. Between-site transmission remains density dependent. Again, we can again obtain expressions for both $R_{0}$ and the equilibrium state.

$$
R_{0}=\frac{\beta}{\gamma+\mu}\left[\rho_{\beta}+\frac{A \Xi}{n \mu}\right] .
$$

Equilibrium $S_{\infty}$ can be given by the positive solution of the following quadratic equation, and from the equation for $\sum_{u} \frac{d i_{u}}{d t}+\frac{d s_{u}}{d t}$ a relationship between $S_{\infty}$ and $I_{\infty}$ is obtained.

$$
\begin{aligned}
0 & =S_{\infty}^{2}(\beta \gamma \Xi / n) \\
& +S_{\infty}\left(\beta \rho_{\beta}(\gamma+\mu)+A \beta \Xi / n-\gamma(\gamma+\mu)\right) \\
& -A(\gamma+\mu) \\
I_{\infty} & =\frac{A-\mu S_{\infty}}{\gamma+\mu}
\end{aligned}
$$

\subsection{Between-site transmission}

We must now specify the form of $\xi_{u, v}$. Reasonable assumptions are a) that the infectious agent disperses over time and loses infectivity as it disperses, and b) that it becomes more rarified with distance. We will assume the first process occurs exponentially with decay rate $\lambda>0$, and the second according to an inverse law, as might be expected if the infectious agent disperses through surface waters. Where the matrix of effective inter-site distances is denoted by $d_{u, v}$, one obtains

$$
\xi_{u, v}=\frac{d_{0}}{d_{u, v}} \exp \left(\lambda\left(d_{0}-d_{u, v}\right)\right)
$$

where $d_{0}$ is a constant parameter representing the distance at which $\xi=1$, which can be considered as representative of farm size. Modelled decay of infectivity is therefore faster than exponential, but becomes closer to exponential at longer distances. The matrix $d$ must now be provided, which will be dependent on the topography of the area studied and the positioning of sites within it. We assume here that the landscape consists of a circular 'island' with sites evenly spaced around its perimeter, $\frac{D}{n}$ units apart, where $D$ is the island circumference. Distance $d_{u, v}$ is then given by whichever is the smaller of $\frac{D}{n}|u-v|$, $\frac{D}{n}|u-v-n|$ and $\frac{D}{n}|u-v+n|$. Therefore, as $n$ decreases, the industry scales towards fewer sites, more widely spaced, though with a proportionately greater density of fish per site.

\subsection{Model simulation}

The deterministic model as presented above may be criticised for its allowing continuous numbers of infected fish. This results in tiny fractional parts of an infected individual being instantaneously produced across all sites, giving rise to epidemics in all sites, even though the risk of between-site infection may be minimal for high $D$ and $\lambda$, and low $n$. As an alternative, the model was simulated using a continuous-time, individual-based, stochastic implementation using a Gillespie-type algorithm (Gillespie, 1977), programmed using $\mathrm{C}++$. At time $t$, the next event to occur is chosen from the $\mathcal{E}=4 n$ possibilities of the types $S \rightarrow I$ (infection), $I \rightarrow \emptyset$ (removal/harvesting), $S \rightarrow \emptyset$ (harvesting) and $\emptyset \rightarrow S$ (stocking), where $\emptyset$ denotes no individual. The $\mathcal{E}$ transition rates $p_{i}$ are then identified from the terms of (2) and (10). The next event occurs after an exponentially distributed time interval

$\Delta t=-\ln ($ Uniform $(0,1)) / \sum_{i=1}^{\mathcal{E}} p_{i}$ where Uniform $(\cdot)$ returns a deviate chosen from the uniform distribution. The event chosen is selected at random, weighting by the rates $p$. This algorithm is relatively efficient given 
that each transition requires only a single function that is slow to calculate: $\ln (\cdot)$, and two uniform random deviates.

The algorithm used is fast, but could not be plausibly applied to a production of 130000 tonnes of fish (Smith, 2008). For strategic purposes, we therefore restrict analysis to a smaller population of up to $10^{5}$ individuals $\left(A / \mu=10^{5}\right)$. To obtain compatible population dynamics in the single-patch model upon disease introduction for the two contact models, $\rho_{\beta}=A / \mu$. Results from the deterministic model, simply solving the differential equations numerically with small step size, are presented for comparison. For ease of reading (to avoid very large and small numbers), parameters for the stochastic model are presented with the following multipliers: $A \times 10^{-5} ; \beta \times 10^{5} ; \rho_{\beta} \times 10^{-5}$. Rescaling parameters as such has no effect on the dynamics of the model. A summary of the model parameters suitable for sensitivity analysis is shown in Table 1 . The model was written using $\mathrm{C}++$.

\section{Results}

\subsection{Sensitivity analysis}

Epidemic time series for the single-patch deterministic model with density dependence are shown in Fig. 1, starting with a non-zero value for $I$. Infection begins to increase only once $S$ reaches a sufficient level for $R_{0}$ to exceed unity. Thereafter, infection depresses $S$ below its disease-free equilibrium level to $S_{\infty}$ and $I$ reaches an equilibrium level $I_{\infty}$. Equilibrium $S_{\infty}$ is not dependent on stocking rate $A$, though proportional prevalence is. With frequency dependence, the epidemic threshold is independent of $S$, and therefore of $A$.

Considering the multiple-site model, behaviour for different $n$ shows potential difference between the frequency-dependent and density-dependent formulations of the model. Maximum potential between-site spread occurs where $(1+\Xi)=n$. Here, the density-dependent model simplifies to a single-patch model, as expected. For other $n$, both $R_{0}$ and equilibrium incidence are dependent on the ratio $\frac{1+\Xi}{n}(6),(8)$. This ratio will vary with $n$ in a manner dependent on the formulation of (14): Calculation of $R_{0}$ for varied $\lambda$ and $n$ with exponential decay of infectivity with distance (14) shows that in all cases, increased $n$ or $\lambda$ leads to decreased $R_{0}$ for the density-dependent model (Fig. 2). However, with frequency dependence, the sensitivity of $R_{0}$ to $n$ is much lower, $n$ only affecting that term containing $\Xi$ (11).

Naïvely, one would assume this means there is no benefit to concentrating output to fewer larger farms, however this does not take into consideration the speed of transmission of disease between such larger, more dispersed farms. Practically, this is of greater importance than equilibrium prevalence as it is hoped human intervention will alleviate an epidemic before that point is reached. This speed can be measured as the degree to which initial infection in the stochastic model spreads around the 'ring' of modelled sites, as shown in Fig. 3, and is dependent upon both $n$ and $\lambda$. In this chart, spikes of infection before equilibrium can be seen for each new infection of a site. This concentration of infection might be expected to increase significantly the probability of neighbouring sites becoming infected during the 'spike'. In this case, the distribution of intervals between successive new infections in Fig. 3 might be expected to be overdispersed with respect to a Poisson process. However, if such an effect exists in the model, it is not sufficiently large in size to cause a significant departure from an exponential distribution (Kolmogorov-Smirnov test: $D=0.037$, $p=0.50$ with $n=498$ simulated intervals).

The long-term equilibrium behaviour of the stochastic model in terms of numbers of susceptibles and infecteds matched closely to the deterministic model where between-site transmission rates are sufficient to disturb the disease-free equilbrium state at the site level, leading to infection across all sites.

Repeat simulations were performed 
for up to 50 time units for both density- and frequency-dependent models with varied $n$ and $\lambda$ (Fig. 4). Higher $\lambda$ implies a quicker decay of infectivity with inter-site distance. As expected from (6) and (11), increasing $n$ causes a greater reduction in epidemic size in the density-dependent model. The number of infected sites at time 50 and the total infected population at this time closely match each other (Fig. 4). Higher $\lambda$ also leads to smaller epidemics to a greater extent than is shown by analysis of the $R_{0}$ equations alone, with a relatively sharp transition in all cases. Each of the lower plateaux of prevelance visible on Fig. 4 represents the epidemic at equilibrium within one site, with no infection having reached any other site over the 50 time units.

There is also interaction between the two parameters $n$ and $\lambda$ : transition to low between-site spread occurs at lower $\lambda$ for lower $n$. This results from the shorter inter-site distance for higher $n$ allowing infection to more readily spread at higher $\lambda$. This interaction is particularly complex for density dependence, where epidemics can persist at the highest $\lambda$ for intermediate $n$. This result is produced by the epidemic failing to spread with low $n$ due to large inter-site distance, but failing to persist within-site for high $n$ due to division into smaller populations.

A slice through Fig. 4 viewed over time with frequency dependence, rather than at a single time point at 50 units, is shown in Fig. 5. As in Fig. 1, those epidemics which spread fastest deplete the larger initial pool of susceptibles, overshooting their eventual equilibrium. Sensitivity to the value of $\lambda$ was large, explaining the rapid drop in sites infected in Fig. 4.

Equations (6) and (11) show that $R_{0}$ in the density-dependent model is completely correlated with $A$, and partly so in the frequency dependent model. The epidemic threshold occurs at $R_{0}=1$. This is explored further in Fig. 6 where $A$ and $n$ are varied, and the same results recorded as in Fig. 2. For the parameter values used $\left(\lambda=0.5, D=100, d_{0}=\mu=1, \gamma=2\right.$, $\beta=40$ ), the epidemic threshold occured at $A=0.3$ and $A=0.9$ for $n=4$ and $n=12$ respectively. However, as is typical with SIR or SIS-type models, numbers of infected cases just above the threshold are small.

\subsection{Effect of the transmission kernel}

We make no attempt to simulate an industry-wide epidemic of ISA or IPN here, particularly in the absence of an estimate for $\beta$. However, there is utility in estimating the relative sensitivity of the between-site force of infection kernel $\xi_{v, u}$ between infected and susceptible sites, which is inversely proportional to the time to infection between sites. This is carried out for the nearest pair of sites only, considered as being distance $d_{1}$ apart. Inference of the bounds of the infection kernel are derived from literature estimates (Jarp and Karlsen, 1997; Murray et al., 2005; Scheel et al., 2007).

Murray et. al. (2005) used a particle tracking model with parameterisation for two specific diseases: infectious pancreatic necrosis virus (IPN) and ISA, appropriate for Atlantic salmon farms in Scotland. These authors predicted a drop in relative concentration of infectivity of $\times 1000$ over distances of approximately 8 to $20 \mathrm{~km}$ for ISA, and over distances of 10 to $90 \mathrm{~km}$ for IPN for current speeds of 1 to $8 \mathrm{~cm} \mathrm{~s}^{-1}$, with IPN being a more robust virus. In comparison, for ISA, Scheel et al. (2007) fit a model giving decreased infection with distance proportional to $\exp \left(-0.415 d_{u, v}\right)$, which suggests a decay of infectivity of $\times 1000$ over a distance of $16.7 \mathrm{~km}$, within the range of Murray et al. (2005). This comparatively local spread for ISA is in agreement with Jarp and Karlsen (1997), who found that approximately $50 \%$ of ISA cases in Norwegian sea sites were within 10 $\mathrm{km}$ of other ISA positive sites. Scheel et al. (2007) suggest that the risk for ISA of being in the same local contact network is equal to that due to seaway distance at a distance of $5 \mathrm{~km}$. We use these data to parameterise the transmission kernel. 
From Scheel et al. (2007), we assume an effective farm size at the distance where local contact is equivalent to seaway distance: $d_{0}=5 \mathrm{~km}$. We then re-express equation (14) into the following form, where $\phi$ represents a proportional drop in infectivity due to the distance kernel over the distance $d_{1}-d_{0}$ :

$$
\phi=\frac{d_{0}}{d_{1}} \exp \left(\lambda\left(d_{0}-d_{1}\right)\right)
$$

A caveat here is that our model assumes faster than exponential decay at short distances, and therefore $\xi$ is somewhat sensitive to the values of $d_{1}$ and $d_{0}$ as well as their difference.

$$
\text { Setting } \phi=0.001, d_{0}=5 \mathrm{~km} \text { and }
$$
using the ranges of $d_{0}-d_{1}$ given above, the corresponding ranges of values of $\lambda$ appropriate for the two diseases were obtained. These ranges were $0.98>\lambda>0.43$ for ISA and $0.80>\lambda>0.11$ for IPN, covering a wide range of the parameter values explored in the sensitivity analysis above. The implications of this upon relative transmission rate $\xi$ for varied nearest distance between sites is shown in Table 2. It shows that aggregating production is likely to have a protective effect against ISA for inter-site spread, though for IPN, the sign of the effect is determined by the expected current speed, at worst weakly increasing risk.

\section{Discussion}

\subsection{Model behaviour and assumptions}

This model demonstrates, in a practical sense, three distinct states: First, disease-free equilibrium; second, epidemic within a single site; and third, spread amongst multiple sites. These last two are not mathematically distinct given that with enough time, the epidemic will spread to other sites unless $\Xi=0$.

Our model results suggest that whether aggregation of production benefits or hinders disease control depends upon the disease parameters, in particular, on the form of the model in terms of density or frequency dependence. Aggregating production has a negative effect through increasing local host density under density dependence, but this effect is absent (within site) under frequency dependence. However, for both density and frequency dependence, causing 'firebreaks' in the industry has a positive effect on reducing the number of infected individuals and sites under more aggregated production. The effectiveness of this however does vary according to the dispersal distance of the pathogen (controlled by $\lambda$ ): where infectivity declines fast with distance, aggregation of production more effectively prevents between-site infection. For density dependence, the model also provides a warning that a small increase in production can tip the balance between $R_{0}<1$ and $R_{0}>1$.

Density dependence and frequency dependence are two opposing model paradigms. Which is appropriate depends on the disease and its transmission mechanism. Where transmission is due to direct contact, and contact rate is independent of the density of fish, then a frequency dependent mechanism may be most apt, as might also be the case for STIs in some human populations. Where infectivity is dispersed within the water column, a density dependent model may be more suitable. Even here, however, shoaling fish might maintain a density that is not directly proportional to their number in a fixed volume. For example, Atlantic salmon have been shown to maintain an effective density higher than calculated density, to an extent determined by environmental conditions such as lighting (Juell et al., 2003). For wild fish stocks, social structure is particularly complex, with schools themelves clustered spatially (Haugland and Misund, 2004). Under density dependence, disease transmission becomes more difficult under rarified conditions, akin to the ecological concept of the Allee effect. However, in reality, diseases may lie at an intermediate 
point on the density dependence-frequency dependence spectrum.

A simple framework for modelling the decay of infectivity with distance, the exponential distribution, is used. This distribution is tunable through its parameter $\lambda$ to account for diseases whose infectious agent is viable in water for greater or lesser time periods. A reciprocal function is also used to model the geometrical dispersion of the infectious agent over a wider ring with increasing distance, but compared with this, the exponential distribution has a smaller tail, producing considerably less infection at longer distances. However, diffusion, advection, and a non-exponential decay in infectivity may produce a decay with distance in infectivity with either larger or smaller tails than modelled here.

The model also assumes that risk of infection is linearly proportional to amount of infectious agent present. Where there is a minimum infectious dose of pathogen, for example due to the requirement to overcome an innate immune system, the model will therefore tend to overpredict the risk of infection at long distance. The effective decay of infectivity with distance will not only be determined by tidal speed, but also by temperature and salinity, the effects of which are different on different viruses (Toranzo \& Hetrick, 1982; Hawley \& Garver, 2008). The stochastic Gillespie algorithm used here is helpful for modelling such threshold behaviour. It is easy to implement, but not fast enough to model an entire industry, fish by fish. Were this required, amended algorithms could be used, for example by using the Gillespie algorithm where numbers of infected individuals on a site are small, and conventional mass-action models where infected individuals are large in number and these models are an appropriate approximation.

The transmission kernel assumes that the force of infection between sites is related to distance. However, there is likely to be both heterogeneity in susceptibility and infectivity both within and between sites, and other routes of infection than local spread. Inter-site contact can be represented by a network, where the direction and strength of potentially infectious contact by mechanisms such as fish movements is explicitly modelled between individual pairs of farms. This has been developed for UK aquaculture industries by Thrush and Peeler (2006), Munro and Gregory (2009) and Green et al. (2009). Network spread tends to reduce the effective distance between farms compared to local spread, due to long-distance connections producing 'small world'-like network dynamics (Kao et al., 2006). However, contact between marine salmon sites due to movements is relatively low (Munro et al. 2009) - though an important potential route for disease introduction - and therefore there remains utility in modelling the shorter-timescale spread of disease directly through local spread.

A further modelling assumption is that of not incorporating culling and fallow periods. Once infected, sites remain infected excepting the remote likelihood of stochastic extinction. Fallow periods would return sites to the susceptible state, after fallowing. A particular extension to the model presented here would be to incorporate synchronised fallowing periods where stocking and harvesting are synchronised within a particular area. Modelling the behavioural or societal response to disease or disease control measures is difficult. It has been assumed above that the disease to be modelled is not so economically damaging that on-site infection leads to immediate imposition of control measures sufficiently draconian as to remove the onward spread of all disease. Also, our model is more appropriate for diseases such as IPN or ISA that may spread at the within-farm level comparatively quickly compared with the time-span of stocking and culling.

\subsection{Practical application of the model}

As presented above, our model is a theoretical framework that can be, through 
sensitivity analysis, used strategically to identify broadly under what conditions aggregating or distributing production helps or hinders the control of different diseases. However, before the model can be used to model a specific disease problem over a region, further disease- and site-specific data are required for parameterisation, as summarised in Table 3.

A potential means for partial parameterisation of the connectivity between sites $\xi$ is the output from advection models. Such models (Murray et al., 2005; Murray and Gillibrand, 2006) simulate the flow of infectivity between sites due to water currents. Aside from the complication of the delay between shedding of infectious agent on one site, and its arrival at another site, the weighting and directionality of such potentially infectious contact, as predicted by advection models, could again be incorporated into existing contact network models for disease transmission. Moreover, anthropogenic spread of disease through, for example, live fish movements can also be incorporated into a network model framework (inter alia Green et al., 2009), and is a natural extension to the strategic model presented here.

Advection-diffusion particle-tracking models explicitly account for the delay between shedding of infectious agent from one site, and the arrival of infectious agent at another, a feature which is not incorporated into our model framework here. However where travel times between sites subject to inter-site infection are short, then this is a reasonable approximation. Timing could be incorporated by introducing a lag term:

$$
\dot{i_{u}}=\ldots \beta s_{u} \sum_{v=1}^{n} \int_{0}^{\infty} \xi_{v, u}(\tau) i_{v}(t-\tau) d \tau \ldots .
$$

Here, $\xi$ is time dependent and this kernel function plus source farm infectivity at time $\tau$ before the present $t, i_{v}(t-\tau)$, determine infection rate, allowing for diffusion of infectivity over a period of specified length. The required kernel functions $\xi_{v, u}(\tau)$ could be derived as output from the advection models, where these could be parameterised for specific farms.

\section{Acknowledgements}

This work was made possible by co-funding through Fisheries Research Services, Aberdeen. With thanks to Alexander Murray and Jimmy Turnbull for helpful discussion about matters arising in this paper.

\section{References}

Anon, 2008. Untitled. http://www.frs-scotland.gov.uk/ FRS.Web/Uploads/Documents/ Management_Area_Maps_August08.pdf Accessed 27 March 2009.

Fulford, G.R., Roberts, M.G., Heesterbeek, J.A.P., 2002. The metapopulation dynamics of an infectious disease: tuberculosis in possums. Theor. Pop. Biol. 61, 15 - 29.

Gillespie, D.T., 1977. Stochastic simulations of coupled chemical reactions. J. Phys. Chem. 81, 2340 2361.

Green, D.M., Gregory, A., Munro, L.A., 2009. Small- and large-scale network structure of live fish movements in Scotland. Prev. Vet. Med. 91, 261 269.

Grenfell, B., Harwood, J., 1997. (Meta)population dynamics of infectious diseases. TREE 12, 395 399.

Gustafson, L.L., Ellis, S.K., Beattie, M.J., Chang, B.D., Dickey, D.A., Robinson, T.L., Marenghi, F.P., Moffett, P.J., Page, F.H. 2007. Hydrographics and the timing of infectious salmon anemia outbreaks among Atlantic salmon (Salmo salar L.) farms in the Quoddy region of Maine, USA and New Brunswick, Canada. Prev. Vet. Med. $78,35-56$. 
Haugland, E.K., Misund, O.A., 2004. Evidence for a clustered spatial distribution of clupeid fish schools in the Norwegian Sea and off the coast of southwest Africa. ICES J. Marine Sci. 61, 1088 - 1092 .

Hawley, L.M., Garver, K.A., 2008. Stability of viral hemorrhagic septicemia virus (VHSV) in freshwater and seawater at various temperatures. Dis. Aquat. Org. $82,171-178$.

Høgåsen, H.R.,, Brun, E., 2003. Risk of inter-river transmission of Gyrodactylus salaris by migrating Atlantic salmon smolts, estimated by Monte Carlo simulation. Dis. Aquat. Org. 57, 247 254.

Jarp, J., Karlsen, E., 1997. Infectious salmon anaemia (ISA) risk factors in sea-cultured Atlantic salmon Salmo salar. Dis. Aquat. Org. 28, $79-86$.

Juell, J.-E., Oppedal, F., Boxaspen, K., Taranger, G.L., 2003. Submerged light increases swimming depth and reduces fish density of Atlantic salmon Salmo salar L. in production cages. Aquaculture Res. 34, 469 - 477.

Kao, R.R., Danon, L., Green, D.M.,, Kiss, I.Z., 2006. Demographic structure and pathogen dynamics on the network of livestock movements in Great Britain. Proc. R. Soc. B 273, 1999 - 2007.

Levins, R., 1969. Some demographic and genetic consequences of environmental heterogeneity for biological control. Bull. Entemol. Soc. Am. 15, 237 - 240.

Mollison, D., 1991. Dependence of epidemic and population velocities on basic parameters. Math. Biosci. 107, $255-287$.

Munro, L., Gregory, A., 2009. Application of network analysis to fish movement data. J. Fish Dis. 32, 641 - 644.
Munro, P.D., Murray, A.G., Fraser, D.A., Peeler, E.J., 2003. An evaluation of the relative risks of infectious salmon anaemia transmission associated with different salmon harvesting methods in Scotland. Ocean and Coastal Management 46, 157 - 174.

Murray, A.G., Amundrud, T.L., Gillibrand, P.A., 2005. Models of hydrodynamic pathogen dispersal affecting Scottish salmon production: modelling shows how Scotland eradicated ISA, but not IPN. Bull. Aquacul. Assoc. Canada 105-1, $79-86$.

Murray, A.G., Gillibrand, P.A., 2006. Modelling salmon lice dispersal in Loch Torridon, Scotland. Marine Pollution Bulletin 53, 128 - 135.

Murray, A.G., Peeler, E.J., 2005. A framework for understanding the potential for emerging diseases in aquaculture. Prev. Vet. Med. 67, 223 235.

Murray, A.G., Smith, R.J., Stagg, R.R., 2002. Shipping and the spread of infectious salmon anemia in Scottish aquaculture. Emerging Infectious Diseases 8, 1 - 5 .

Nicholson, A.J., Bailey, V.A., 1935. The balance of animal populations. Part 1. Proc Zool Soc Lond 3:551 - 598.

Paisley, L.G., Karlsen, E., Jarp, J., Mo, T.A., 1999. A Monte Carol simulation model for assessing the risk of introduction of Gyrodactylus salaris to the Tana river, Norway. Dis. Aquat. Org. 37, 145 152.

Peeler, E.J., Murray, A.G., Thebault, A., Brun, E., Giovaninni, A., Thrush, M.A., 2007. The application of risk analysis in aquatic health management. Prev. Vet. Med. 81, 3- 20.

Reno, P.W., 1998. Factors involved in the dissemination of disease in fish populations. J. Aquat. Anim. Health. $10,160-171$. 
Scheel, I., Aldrin, M., Frigessi, A., Jansen, P.A., 2007. A stochastic model for infectious salmon anemia (ISA) in Atlantic salmon farming. J. R. Soc. Interface 4, $699-706$.

Smith, R.J., 2008. Scottish fish farms: Annual production survey 2007. http://www.frs-scotland.gov.uk/ FRS.Web/Uploads/Documents/ surveytext2007final.pdf Accessed 27 March 2009.

Thrush, M., Peeler, E., 2006. Stochastic simulation of live salmonid movement in England and Wales to predict potential spread of exotic pathogens. Dis. Aqua. Org. 72, $115-123$.

Toranzo, A.E., Hetrick F.M., 1982.

Comparative stability of two salmonid viruses and poliviorus in fresh, estuarine and marine waters. J. Fish Diseases 5, 223 - 231. 
Table 1 Model parameters used to describe the spread of infectious disease in a region of an aquaculture industry. For parameter dimensions, $L$ and $T$ represent length and time, and $M$ the measurement unit of the fish, which could be fish number or biomass.

\begin{tabular}{lll} 
Parameter & & Dimension \\
\hline$\beta$ & force of infection posed by infected fish & {$[M]^{-1}[T]^{-1}$} \\
& (transmission rate) & \\
$\rho_{\beta}$ & population size for equal transmission rate for & {$[M]$} \\
& density- and frequency-dependent models & \\
$\gamma$ & removal rate for infected fish & {$[T]^{-1}$} \\
$\mu$ & harvesting rate for all fish & {$[T]^{-1}$} \\
$A$ & stocking rate of new fish across all sites & {$[M][T]^{-1}$} \\
$n$ & number of sites & {$[1]$} \\
$D$ & linear distance across which sites are spread & {$[L]$} \\
$d_{0}$ & effective farm size (distance at which within- & {$[L]$} \\
& and between-site infection become equal) & \\
$\lambda$ & exponential decay rate of infectivity & {$[L]^{-1}$}
\end{tabular}

Table 2 Sensitivity analysis of a model of the spread of infectious disease in a region of an aquaculture industry. Between-site relative force of infection $\xi$ for varied rate of decay of infectivity with distance $\lambda$ and nearest-neighbour distance $d$. Single-farm densities of fish $S$ and $I$ are assumed inversely proportional to $d$ and a constant disease prevalence is assumed; effective farm size $d_{0}=5 \mathrm{~km}$.

\begin{tabular}{lllll}
$d, \mathrm{~km}$ & \multicolumn{5}{c}{$\lambda, \mathrm{km}^{-1}$} \\
& IPN range & \multicolumn{3}{c}{ ISA range } \\
& 0.11 & 0.8 & 0.43 & 0.98 \\
\hline 5.0 & 1 & 1 & 1 & 1 \\
7.5 & 1.14 & 0.20 & 0.51 & 0.13 \\
10.0 & 1.15 & 0.037 & 0.23 & 0.015 \\
12.5 & 1.10 & 0.0062 & 0.099 & 0.0016 \\
15.0 & 1.00 & 0.0010 & 0.041 & 0.0002
\end{tabular}


Table 3 Data required for parameterisation of a model of the spread of a specific infectious disease in a region of an aquaculture industry.

Site data:

Stocking/ harvesting/ fal- Parameterisation of stocking rate $A$ and harvesting rate $\mu$, low details potentially made time-varying to simulate separable stocking, harvest, and fallow periods.

Farm management/ mor- Parameterisation of removal rate $\gamma$ and harvesting rate $\mu$, tality data potentially time-varying.

Hydrography, georeferenc- Allows calculation of effective site-site distance $d_{u, v}$ and siteing details site transmission rate $\xi_{u, v}$.

Live fish movement data, As a further component of site-site transmission rate $\xi_{u, v}$ for wellboat records, etc. network models.

Disease data:

General literature review Identification of potential transmission routes, etc., parameterisation of transmission rate $\beta$.

Incidence/ prevalence out- Allows fitting of model to estimate unknown disease parambreak data eters.

Longevity studies of infec- Component of site-site transmission rate $\xi_{i, v}$ through kernel tious agent function $\lambda$. 


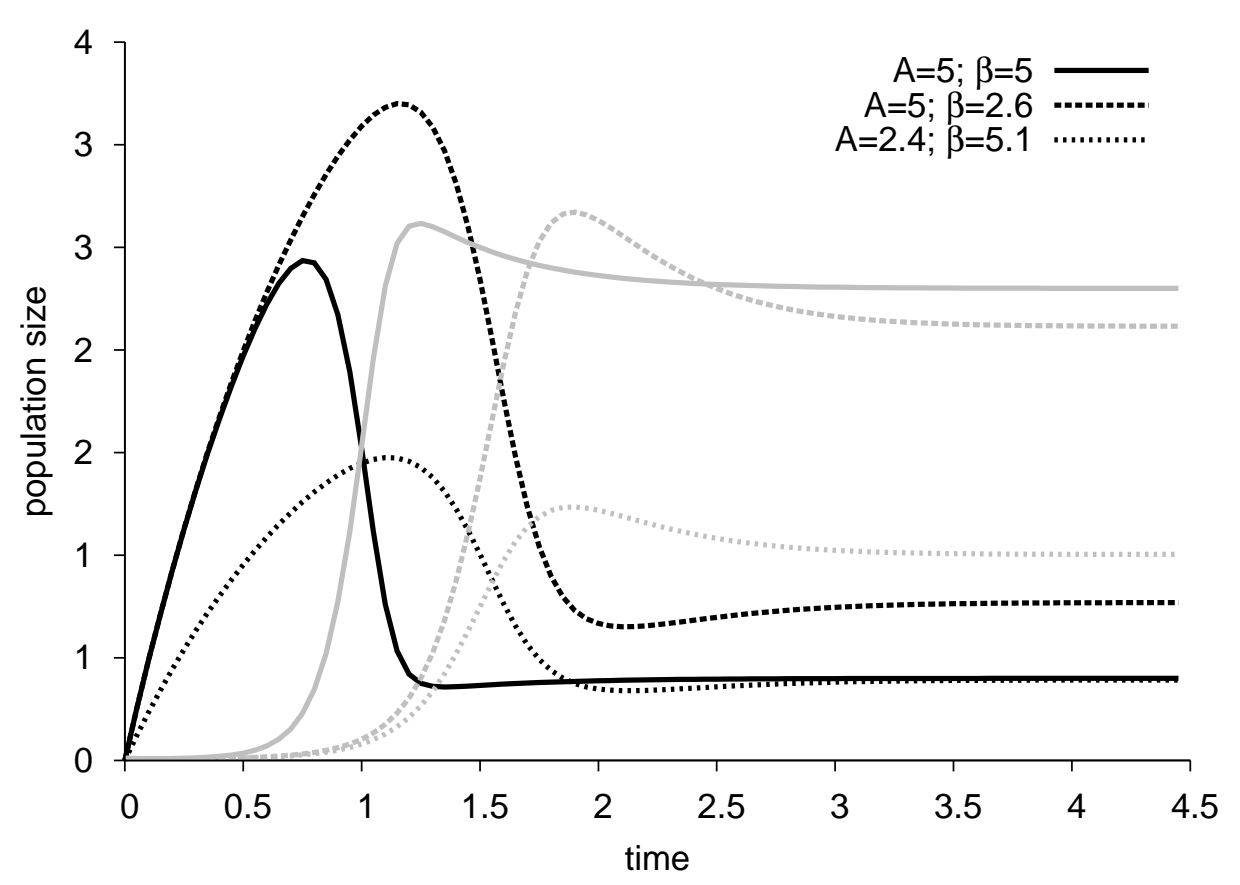

Figure 1: Epidemic dynamics for the single-site density-dependent model with removal rate $\gamma=1$ and harvesting rate $\mu=1$ (deterministic simulation). Populations of susceptible fish $(S)$ and infected fish $(I)$ are shown in black and grey, respectively. Initial populations at the start of the simulation were $I_{0}=0.01$ and $S_{0}=0$. Varied disease transmission rates $\beta$ and total stocking rate $A$ are shown.

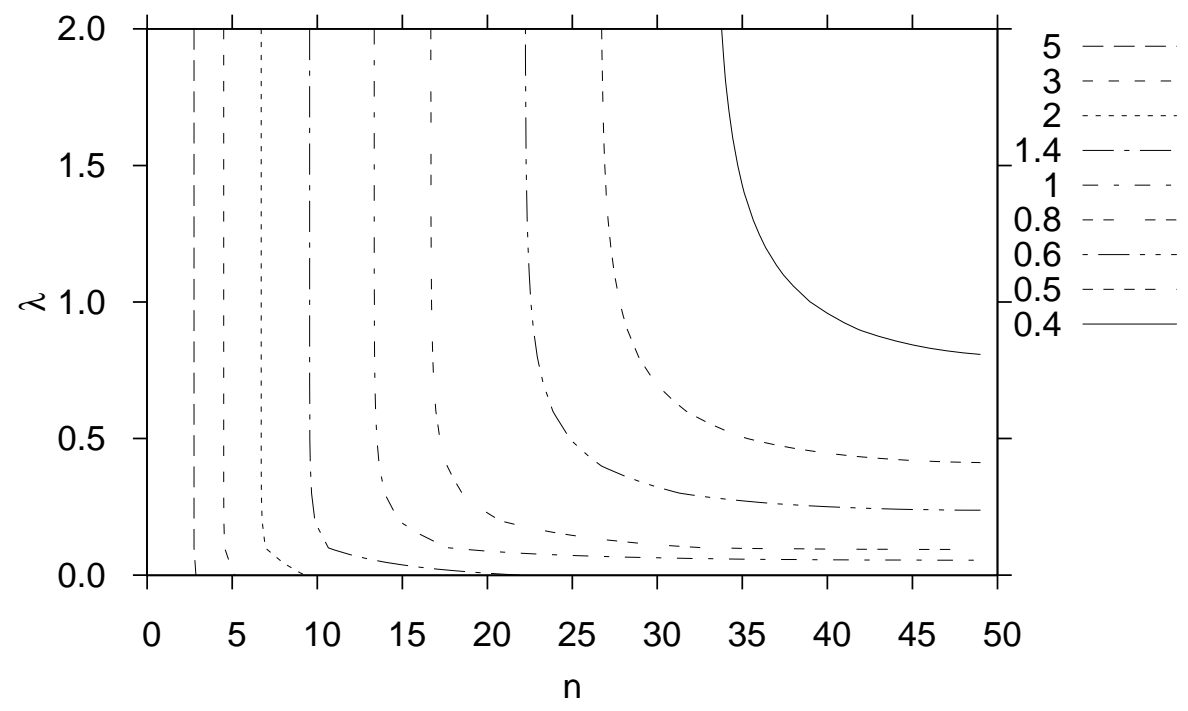

Figure 2: Contour plot of modelled $R_{0}$ for the deterministic, multi-site model with density dependence, versus number of sites $n$ and rate of decay of infectivity with distance $\lambda$. Other parameters were total region size $D=100$; effective farm size $d_{0}=1$; transmission rate $\beta=40$; total stocking rate $A=1$; harvesting rate $\mu=1$; and removal rate $\gamma=2$. 


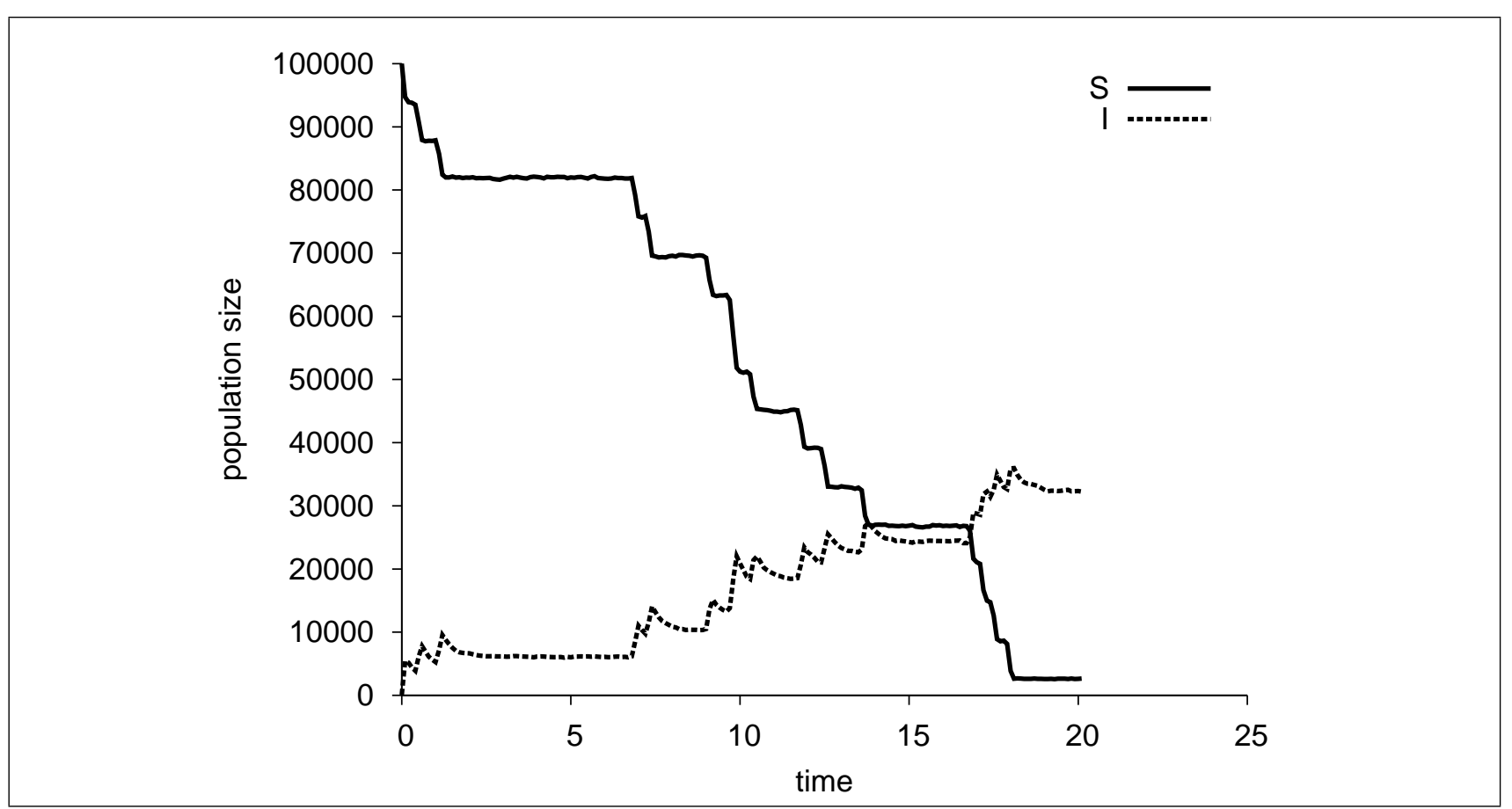

Figure 3: Epidemic dynamics for $n=16$ sites for a single run of the frequency-dependent stochastic model, demonstrating stochastic spread between sites leading to successive spikes of infection, returning to new equilibria. Parameters as in Fig. 2 with rate of decay of infectivity with distance $\lambda=1.5$ and equivalent transmission rate to the density-dependent model at $\rho_{\beta}=1$. 

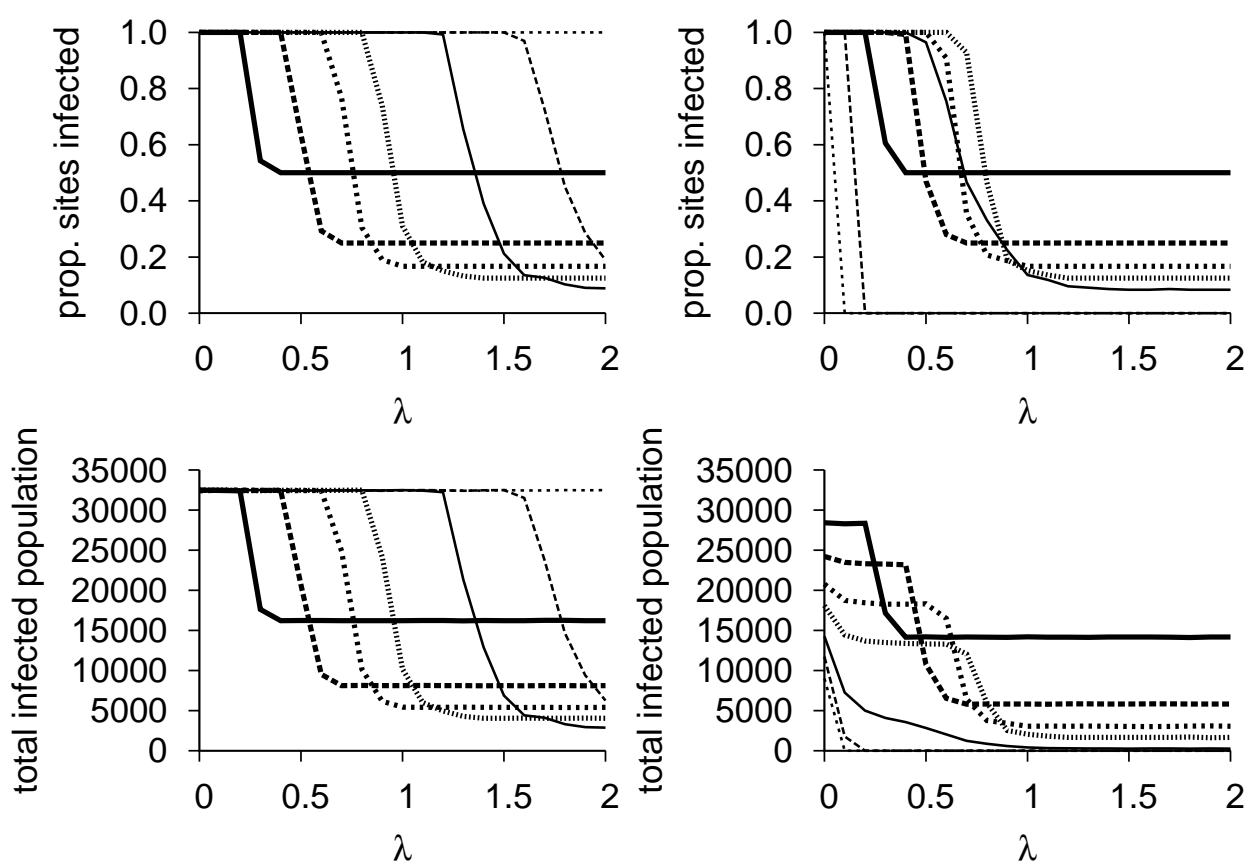

Figure 4: Proportion of sites infected and the total infected population after 50 time units for the stochastic, multi-site model, versus number of sites $n$ and decay of infectivity with distance $\lambda$. Parameters as in Fig. 2 (except $\lambda$ ) and transmission rates in the two models were equivalent at $\rho_{\beta}=1$. Number of sites $n$ is varied as 2, thick solid; 4, long dash; 6, medium dash; 8, short dash; 12 , thin solid; 16 , long dash; 24, medium dash. Left: frequency dependent model; right: density dependent model. Means of 25 model runs are shown for each point. 


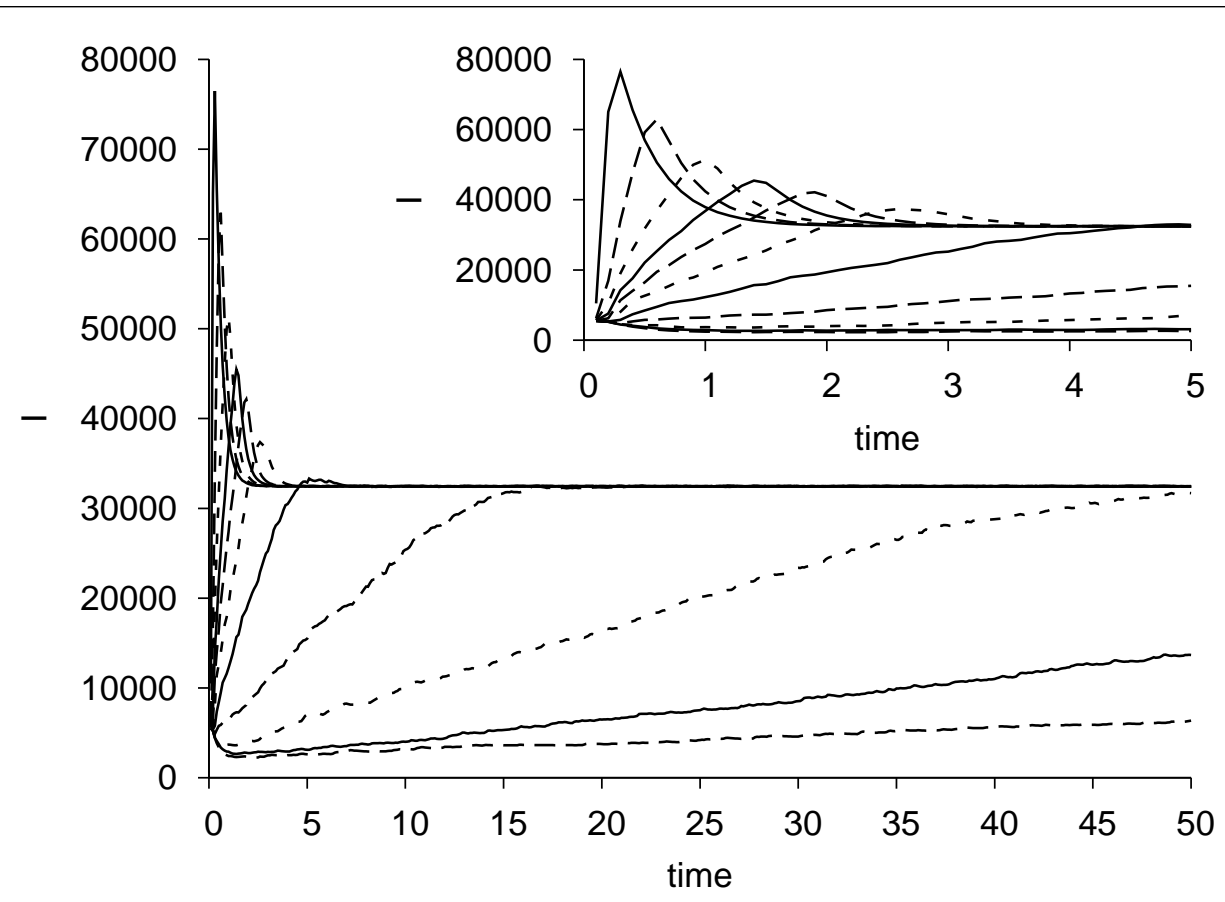

Figure 5: Time series for infected number for the stochastic, multi-site model with frequency dependence for different values of the rate of decay of infectivity with distance $\lambda$. Up to 50 time units are shown, with up to 5 time units inset. Parameters as in Fig. 2 with equivalent transmission rate to the density-dependent model at $\rho_{\beta}=1$ and $n=16$ sites. The values of $\lambda$ vary across series from 0.0 to 2.0 with step-size 0.2 from the fastest to slowest spread simulations. Means of 16 simulations are shown. Note that decay of infectivity with distance occurs still at $\lambda=0$ due to the hyperbolic function in (14). 

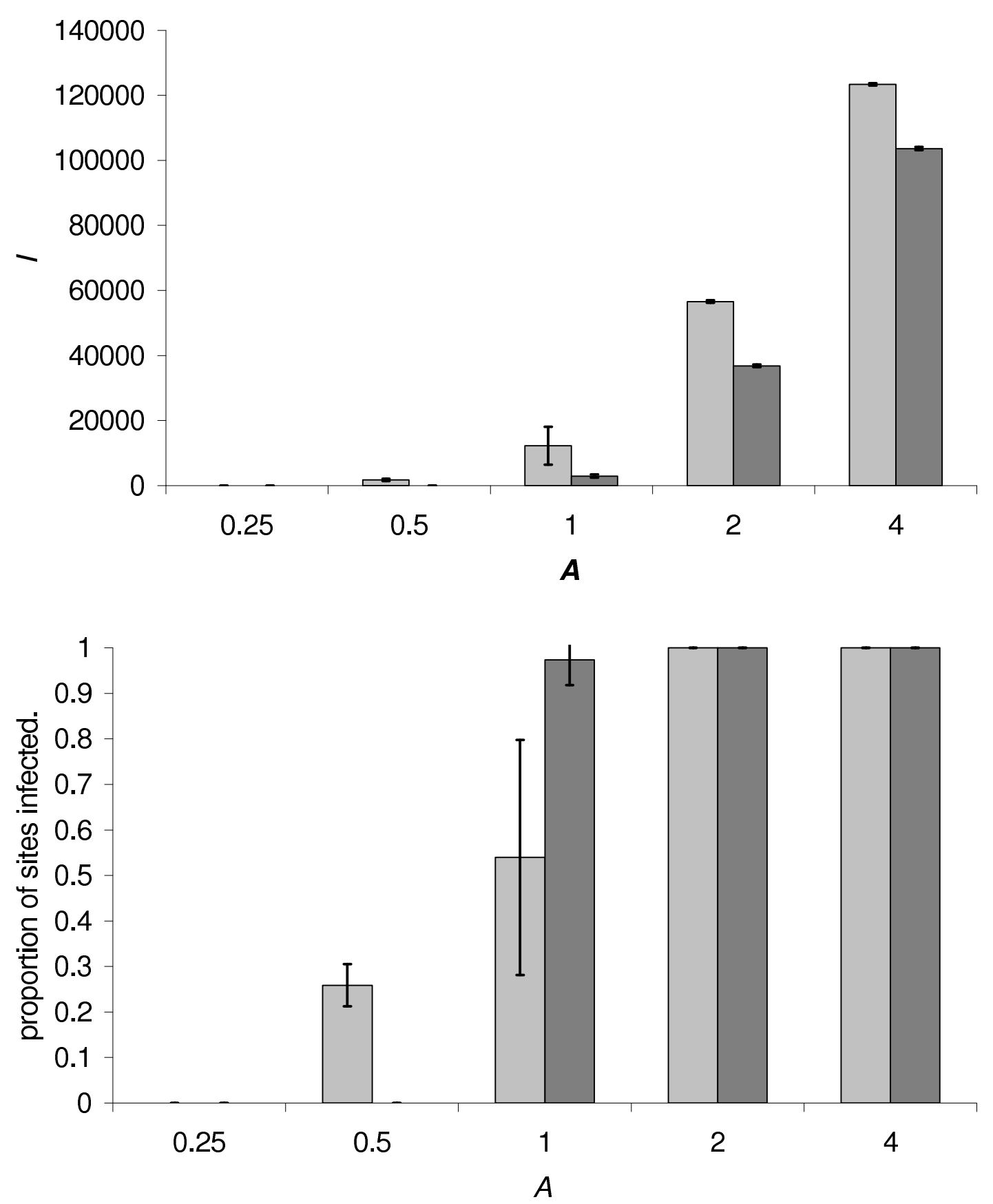

Figure 6: Proportion of sites infected and infected population after 50 time units for the stochastic, multi-site model with density dependence, versus number of sites $n$ and total stocking across all sites A. Parameters as in Fig. 2 except the rate of decay of infectivity with distance parameter $\lambda=0.5$. Light columns: $n=4$; dark columns: $n=12$. Means and standard deviations are shown, based on 50 simulations for each point. 